\title{
COVID-19 and endoscopic management of superficial gastrointes- tinal neoplastic lesions: a multinational cross-sectional survey
}

Authors

Marta Rodríguez-Carrasco ${ }^{1}$, Eduardo Albéniz ${ }^{2}$, Pradeep Bhandari ${ }^{3}$, Torsten Beyna ${ }^{4}$, Michael J. Bourke ${ }^{5}$, Ahyeon Min ${ }^{5}$, Philip W. Y. Chiu ${ }^{6}$, Simon Chu ${ }^{6}$, Hon Chi Yip ${ }^{6}$, Pierre H. Deprez ${ }^{7}$, Fabian Emura ${ }^{8,9}$, Alessandro Repici ${ }^{10}$, Noriko Suzuki ${ }^{11}$, Naohisa Yahagi ${ }^{12}$, Yoko Kubosawa ${ }^{12}$, Cesare Hassan ${ }^{13}$, Mário Dinis-Ribeiro ${ }^{1,14}$

Institutions

1 Department of Gastroenterology, Portuguese Oncology Institute of Porto, Porto, Portugal

2 Endoscopy Unit and Gastroenterology Department, Complejo Hospitalario de Navarra, Navarrabiomed Biomedical Research Center, UPNA, IdiSNA, Pamplona, Spain

3 Department of Gastroenterology, Queen Alexandra Hospital, Portsmouth, UK

4 Department of Gastroenterology, Evangelisches Krankenhaus, Düsseldorf, Germany

5 Department of Gastroenterology and Hepatology, Westmead Hospital, Sydney, Australia

6 Endoscopy Center, Institute of Digestive Disease, The Chinese University of Hong Kong, Hong Kong, China

7 Department of Hepato-Gastroenterology, Cliniques universitaires St-Luc, Université Catholique de Louvain, Brussels, Belgium

8 EmuraCenter Latinoamérica, Bogota, Colombia

9 Gastroenterology Division, Universidad de La Sabana, Chia, Colombia

10 Department of Gastroenterology, Humanitas Research Hospital, Humanitas University, Rozzano, Milan, Italy

11 Wolfson Department of Endoscopy, St Mark's Hospital, London, UK

12 Division of Research and Development for Minimally Invasive Treatment, Cancer Center, Keio University School of Medicine, Tokyo, Japan

13 Department of Gastroenterology, Nuovo Regina Margherita Hospital, Rome, Italy

14 MEDCIDS - Department of Community Medicine, Health Information and Decision, Faculty of Medicine, University of Porto, Porto, Portugal

submitted 23.5.2020

accepted after revision 11.8 .2020

published online 11.8 .2020

Bibliography

Endoscopy 2021; 53: 173-177

DOI 10.1055/a-1237-5221

ISSN 0013-726X

(c) 2020. Thieme. All rights reserved.

Georg Thieme Verlag KG, Rüdigerstraße 14,

70469 Stuttgart, Germany
Corresponding author

Mário Dinis-Ribeiro, MD, PhD, Gastroenterology Department, Portuguese Oncology Institute of Porto, Rua Dr. Bernardino de Almeida, 4200-072 Porto, Portugal

Fax: +351-22-5513646

mario.ribeiro@ipoporto.min-saude.pt

$\circledast$ Scan this QR-Code for the author commentary.

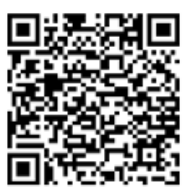

\section{ABSTRACT}

Introduction We aimed to report the impact of the pandemic lockdown period on the treatment and prognosis of superficial gastrointestinal neoplastic lesions.

Methods A survey was completed by 11 centers from four continents regarding postponements during the early lockdown period of the pandemic, and the same period in 2019. Results In 2020, $55 \%$ of the scheduled procedures were deferred, which was 11 times higher than in 2019; the main reasons were directly related to COVID-19. In countries that were highly affected, this proportion rose to $76 \%$ vs. $26 \%$ in those where there was less impact. Despite the absolute reduction, the relative distribution in 2019 vs. 2020 was similar, the only exception being duodenal lesions (affected by a $92 \%$ reduction in mucosectomies). Although it is expected that the majority of postponements will not affect the stage (based on the results from biopsies and/or endoscopic appearance), $3 \%$ of delayed procedures will probably require surgery.

Conclusions The lockdown period caused by the SARSCoV-2 pandemic led to a substantial reduction in the number of endoscopic resections for neoplastic lesions. Nevertheless, based on clinical judgment, the planned median delay will not worsen the prognosis of the affected patients. 
- Table 1 Participating centers and data collection periods.

\begin{tabular}{|l|l|}
\hline Center (city, country) & $\begin{array}{l}\text { Data collection } \\
\text { period }\end{array}$ \\
\hline $\begin{array}{l}\text { Complejo Hospitalario de Navarra } \\
\text { (Pamplona, Spain) }\end{array}$ & 16 March to 15 April \\
\hline $\begin{array}{l}\text { Cliniques universitaires St-Luc (Louvain, } \\
\text { Belgium) }\end{array}$ & 16 March to 15 April \\
\hline $\begin{array}{l}\text { EmuraCenter Latinoamérica (Bogota, } \\
\text { Colombia) }\end{array}$ & 16 March to 15 April \\
\hline $\begin{array}{l}\text { Queen Alexandra Hospital (Portsmouth, UK) } \\
\text { Evangelisches Krankenhaus (Düsseldorf, } \\
\text { Germany) }\end{array}$ & 23 March to 23 April \\
\hline \begin{tabular}{l} 
Institute of Digestive Disease (Hong Kong) \\
\hline St Mark's Hospital (London, UK)
\end{tabular} & 15 March to 15 April \\
\hline Humanitas Research Hospital (Milan, Italy) & 9 April \\
\hline Westmead Hospital (Sydney, Australia) & 23 March to 20 April \\
\hline Keio University Hospital (Tokyo, Japan) & 15 March to 15 April \\
\hline $\begin{array}{l}\text { Portuguese Oncology Institute of Porto } \\
\text { (Porto, Portugal) }\end{array}$ & 18 March to 17 April \\
\hline
\end{tabular}

- Table 2 Endoscopic resections scheduled in 2019 and in 2020, during the first month of lockdown.

\begin{tabular}{|c|c|c|}
\hline & 2019 & 2020 \\
\hline Planned procedures, $\mathrm{n}$ & 368 & 314 \\
\hline $\begin{array}{l}\text { Time from diagnosis to first appointment, } \\
\text { median (range), weeks }\end{array}$ & $6(1-32)$ & $5(2-20)$ \\
\hline Postponed procedures, n (\%) & $19(5)$ & $173(55)$ \\
\hline \multicolumn{3}{|l|}{ Reasons for rescheduling, $\mathrm{n}$} \\
\hline - Lack of timeslot & 6 & 0 \\
\hline - Lack of human resources & 0 & 12 \\
\hline - Other reasons & 13 & 14 \\
\hline - Clinical decision due to covid-19 & $\mathrm{N} / \mathrm{A}$ & 147 \\
\hline - Lack of PPE & $\mathrm{N} / \mathrm{A}$ & 0 \\
\hline $\begin{array}{l}\text { Time to new appointment, median } \\
\text { (range), weeks }\end{array}$ & $4(2-24)^{*}$ & $8(2-24)^{*}$ \\
\hline \multicolumn{3}{|c|}{ More advanced diagnosis at time of procedure expected/observed, $n$} \\
\hline - Endoscopic management & $0 / 16$ & $168 / 16$ \\
\hline - Surgery required & $0 / 1$ & $5 / 0$ \\
\hline \multicolumn{3}{|c|}{$\begin{array}{l}\text { N/A, not applicable; PPE, personal protective equipment. } \\
\text { * One center reported a waiting list of } 24 \text { weeks for one procedure in } 2019 \\
\text { and for six procedures in } 2020 .\end{array}$} \\
\hline
\end{tabular}

\section{Introduction}

On 11 March 2020, the World Health Organization (WHO) declared the outbreak of coronavirus disease 2019 (COVID-19), caused by SARS-CoV-2, a pandemic [1,2], which marked an unprecedented situation with highly restrictive considerations to be adopted by the majority of countries worldwide.

Because gastrointestinal (GI) endoscopy represents a potential source of transmission of SARS-CoV-2 among healthcare professionals [3-5], the European Society of Gastrointestinal Endoscopy (ESGE) recommended postponing or performing elective procedures according to their priority and the COVID19-related morbidity/mortality. In this context, it was suggested that neoplastic lesions harboring high grade dysplasia (HGD)/early cancer should be performed or rescheduled within less than 12 weeks [6].

The aim of this study was to report the impact of the COVID19 pandemic on the endoscopic treatment and prognosis of superficial neoplastic lesions in countries with differing pandemic severities.

\section{Methods}

An online survey was sent to the endoscopy department in 14 high volume centers in 13 countries from four continents in one or two rounds (in case of non-response) to maximize the response rate. We considered "total confirmed cases" in each country as a criterion to invite the participating centers with the aim of obtaining a wide range of impacts of the COVID-19 pandemic.

The survey collected information regarding consecutive schedules and postponements from patients with neoplastic $\mathrm{Gl}$ lesions in which endoscopic resection was considered feasible, without age restrictions. The surveyed period was the first month after the implementation of endoscopic restrictions in each respective lockdown period, and the same month in 2019. The survey was composed of eight domains applied for each Gl location (esophagus/stomach/colon/duodenum): (1) planned endoscopic mucosal resection (EMR) and endoscopic submucosal dissection (ESD); (2) mean time to the first appointment; (3) postponements; (4) planned rescheduling time; (5) reasons for performing; (6) reasons for postponing; (7) expected outcome (based on histopathological analysis from biopsies and/or endoscopic appearance); (8) observed outcome. The collected data were manually retrieved by reviewing in-hospital databases.

Postponements from 2019 vs. 2020 and the impact between countries according to the severity of the pandemic were analyzed. A country was considered to have a severe impact if it was classified among the 15 most affected countries worldwide, in terms of total confirmed cases, on the day after the data collection period closed $[7,8]$.

Quantitative data were summarized using the median and range. The chi-squared test and $t$ test were used to compare the distributions of categorical and continuous variables, respectively. A $P$ value $<0.05$ was considered to be statistically 


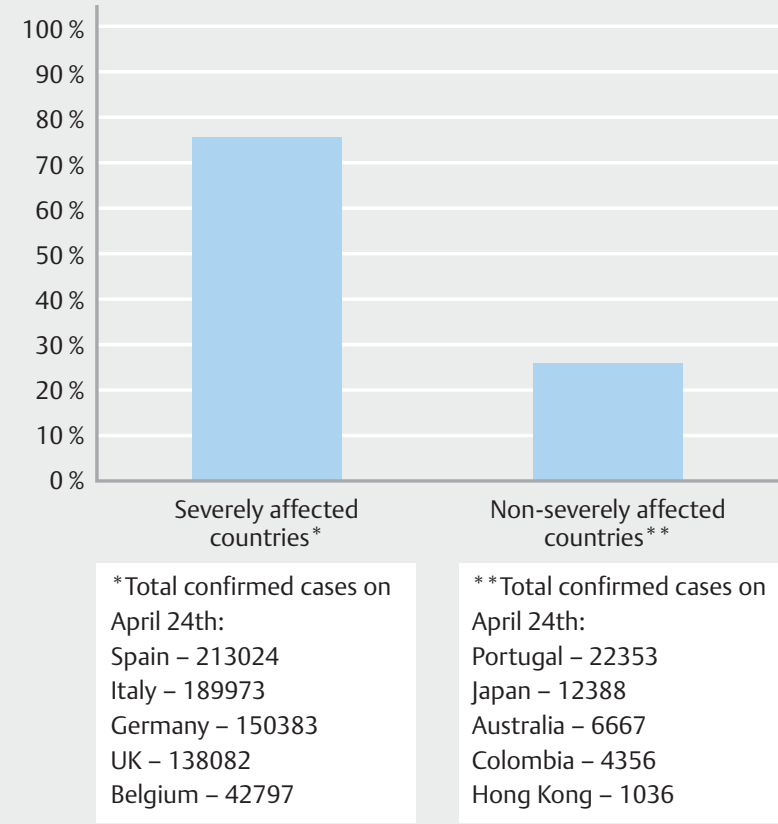

- Fig. 1 Percentage of postponements according to the severity of the COVID-19 pandemic.

significant. Analysis was performed using Excel Office 2019 (Microsoft) and SPSS version 19 software (IBM).

\section{Results}

Among the surveys sent, a response rate of $79 \%$ was obtained. Data from 11 centers in 10 countries across four continents were included. Because of differences in the lockdown dates, the periods analyzed were different in each center and ranged from 15 March to 23 April ( Table 1).

In 2020, $314 \mathrm{Gl}$ lesions were scheduled for resection (EMR/ ESD) during the respective early lockdown periods ( $\triangleright$ Table 2 ). Among them, 141 (45\%) were performed, 56\% by ESD. The presence of $\mathrm{HGD}$ /intramucosal cancer on previous biopsies (59\%) and the availability of personal protective equipment (PPE; $48 \%$ ), were the main reasons to maintain the schedules. In contrast, 173 procedures (55\%) were postponed, mostly owing to a clinical decision related to COVID-19 (86\%) and a lack of resources (human or hospital facilities, due to unit restructuring to attend COVID-19 patients). The majority of postponements $(80 \%)$ were from countries that were severely affected by COVID-19. Moreover, the severity of the pandemic had a substantial influence on the distribution of postponements, which were significantly higher in countries severely affected (76\% vs. $26 \%, P<0.05$ ) ( Fig. 1). In contrast, during the same period of time in 2019, only 19 procedures from a total of 368 were postponed (5\%) (> Table 2 ).

- Fig. 2 represents the comparison per organ and procedure between 2019 and 2020. In 2020, most of the ESDs were gastric and colorectal (47\% and $33 \%$ ), while the vast majority of the
EMRs were performed in the colorectal tract (82\%). The same distribution was observed in 2019 , when $38 \%$ of the ESDs were performed in the stomach, $38 \%$ in the colorectal tract, and most of the EMRs were performed in the colon (70\%). ESDs were more frequently kept and duodenal mucosectomy was the procedure most often rescheduled.

Some missing data have to be considered: first, it was not possible to quantify the exact number of colonic EMRs in one center owing to the high volume of planned procedures; second, during the 2020 lockdown, some patients postponed their procedures through their own choice, making it difficult to retrieve these cases.

Despite the fact that the differences in schedule and rescheduling mean times were not statistically significant, the potential clinical implications of the increase in median rescheduling time in 2020 ( 8 weeks) cannot be ignored as the progression of some lesions may be determined by their histology. One center reported a waiting time of 24 weeks for one procedure in 2019 and for six procedures in 2020. This delay did not affect the estimated staging for the procedure in 2019, but the real impact on these procedures in 2020 is still unknown. Nevertheless, based on histopathological results from biopsies and endoscopic appearance, the authors expect that the majority of the cancelled procedures will not have a negative impact on prognosis caused by reallocation, and only five patients (3\%) will probably require surgery. At the time of concluding this survey, only $9.2 \%$ of the postponed procedures had already been performed, and none of these had required additional elective surgical treatment after histopathological staging.

\section{Discussion}

During the pandemic, hospitals over the world made an enormous effort to provide healthcare to patients affected by COVID-19, while adopting measures to maintain a safe environment for patients and healthcare professionals. As a result, the usual workflow in endoscopy departments changed dramatically, leading to an increase in cancelled procedures [9]. With regard to neoplastic lesions, ESGE recommended maintaining the schedule, if possible, for those lesions harboring HGD/intramucosal cancer (high priority), or rescheduling them within 12 weeks, while low grade dysplasia (low priority) could be rescheduled beyond 12 weeks [6]. However, the impact in terms of cancer progression is unknown.

The present survey aimed to report the effect of lockdown on the endoscopic treatment of neoplastic lesions in countries with differing severities of the pandemic. During lockdown, a dramatic increase in postponed procedures was shown, which was 11 times higher than for the same period in 2019 (55\% vs. $5 \%$ ), with the main reasons being directly related to COVID-19 (clinical decisions and lack of resources). The proportion of procedures rescheduled was significantly influenced by the impact of the pandemic in each country, and the majority of postponements $(80 \%)$ were concentrated in the most severely affected countries. 


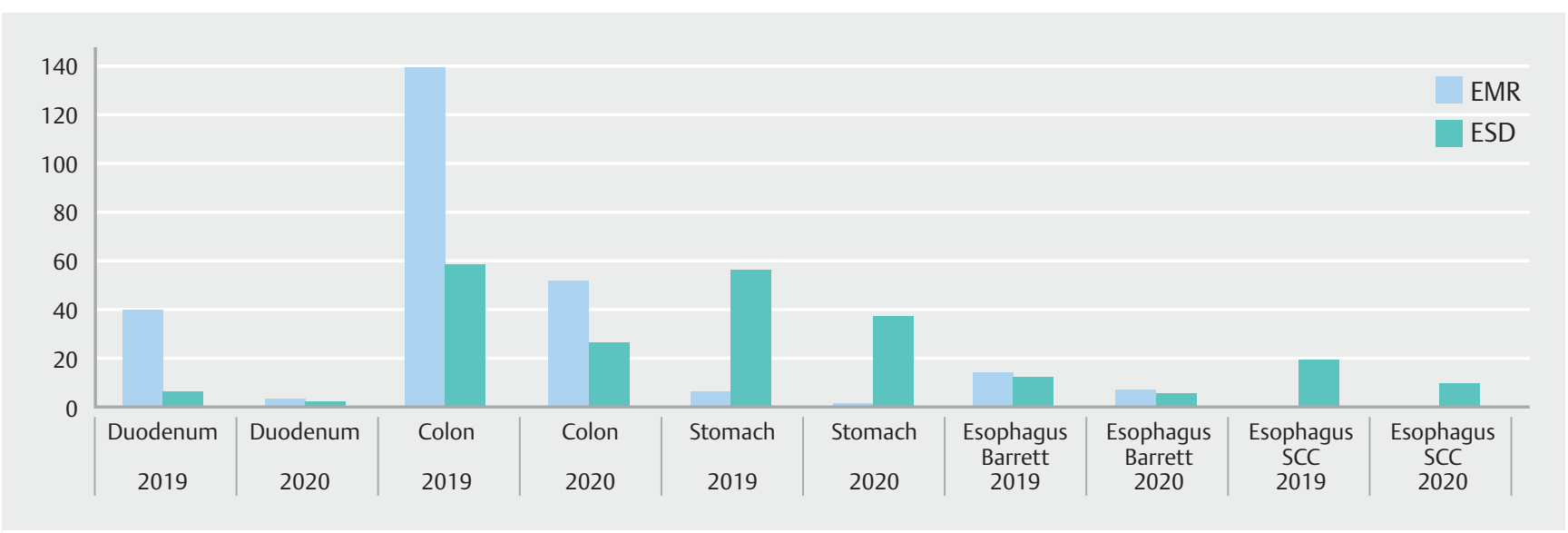

- Fig. 2 Endoscopic procedures performed in 2020 and 2019 (period of time equivalent to the 2020 lockdown). EMR, endoscopic mucosal resection; ESD, endoscopic submucosal dissection; SCC, squamous cell carcinoma.

The most reassuring result is that the main reason to plan the procedure, despite the lockdown, was the presence of HGD/intramucosal cancer, suggesting a hierarchy between the severity of the neoplastic disease and the infective risk for the patient coming to the hospital. The second result is the selective postponing of virtually all of the duodenal procedures. This may be related to two main factors: first, neoplastic duodenal lesions progress slowly and very infrequently present with an already invasive cancer; second, they carry a high risk of severe adverse events that might require admission to an intensive care unit already overfilled with COVID-19 patients. Not unexpectedly, in absolute terms, the largest contribution to the postponement of endoscopic resections came from EMR in the colorectal tract. This may be attributed to the possibility of accurately predicting the risk of invasive cancer simply through a classification based on morphology and location.

The mean planned timing for the performance of delayed endoscopies was within the ESGE recommendations $(<12$ weeks) and, according to observers, it is not expected that the delay will affect the prognosis in the majority of lesions (97\%). However, the real impact is still unknown as most of the procedures have not yet been performed. For this reason, a complete personal history of the patient, including all the documentation, possibly with pictures or videos of the index examination, is critical in deciding when to postpone an endoscopic resection, and the supervision of a senior endoscopist should always be sought for this activity.

Some limitations have to be considered. First, missing data must be considered when interpreting the results because it affected the obtained "n," making it smaller than the real one. Second, the real impact of the waiting time is still unknown as, at the time of completing this manuscript, the pandemic is unpredictable, the majority of postponed procedures have not yet been performed, and histopathological results from those already done are still unavailable. Third, the constant and rapid changing of this pandemic may represent a limitation of the subclassification into severely and non-severely affected coun- tries, so its interpretation should be contextualized within the surveyed period.

To conclude, the SARS-CoV-2 pandemic considerably changed the usual workflow of endoscopy units, increasing the number of cancelled procedures, especially in those countries seriously affected by the pandemic. The timing for rescheduling seems to be optimal and, although the real influence on staging is still unknown, it is expected this will be unaffected.

\section{Competing interests}

F. Emura received research support from Fujifilm. The remaining authors declare that they have no conflict of interest.

\section{References}

[1] WHO Director-General's opening remarks at the media briefing on COVID-19. Available at (Accessed 2 September 2020): https://www. who.int/dg/speeches/detail/who-director-general-s-opening-remarks-at-the-media-briefing-on-covid-19-29-april-2020

[2] Heymann DL, Shindo N. WHO Scientific and Technical Advisory Group for Infectious Hazards. COVID-19: What is next for public health? Lancet 2020; 395: 542-545

[3] Zhang $\mathrm{H}$, Kang Z, Gong $\mathrm{H}$ et al. Digestive system is a potential route of COVID-19: an analysis of single-cell coexpression pattern of key proteins in viral entry process. Gut 2020: doi:10.1136/ gutjnl-2020320953

[4] Lin L, Jiangx X, Zhang Z et al. Gastrointestinal symptoms of 95 cases with SARS-CoV-2 infection. Gut 2020; 69: 997-1001

[5] Gu J, Han B, Wang J. COVID-19: Gastrointestinal manifestations and potential fecal-oral transmission. Gastroenterology 2020; 158: 15181519

[6] Gralnek IM, Hassan C, Beilenhoff U et al. ESGE and ESGENA Position Statement on Gastrointestinal Endoscopy and the COVID-19 Pandemic. Endoscopy 2020; 52: 483-490 
[7] WHO situation report - 95. Available at (Accessed 2 September 2020): www.who.int/docs/default-source/coronaviruse/situation-reports/20200424-sitrep-95-covid-19.pdf?sfvrsn=e8065831_4

[8] The Government of the Hong Kong Special Administrative Region. Latest Situation of Coronavirus Disease (COVID-19) in Hong Kong.
Available at (Accessed: 4 September 2020): https://chp-dashboard. geodata.gov.hk/covid-19/en.html

[9] Repici A, Pace F, Gabbiadini R et al. Endoscopy units and the COVID-19 outbreak: a multi-center experience from Italy. Gastroenterology 2020; 159: 363-366 